3. Calfee DP, Farr BM. Comparison of four antiseptic preparations for skin in the prevention of contamination of percutaneously drawn blood cultures: a randomized trial. J Clin Microbiol 2002;40(5):1660-1665.

4. Little JR, Murray PR, Traynor PS, Spitznagel E. A randomized trial of povidone-iodine compared with iodine tincture for venipuncture site disinfection: effects on rates of blood culture contamination. Am J Med 1999;107(2):119-125.

5. Strand CL, Wajsbort RR, Sturmann K. Effect of iodophor vs iodine tincture skin preparation on blood culture contamination rate. JAMA 1993 269(8):1004-1006.

6. Kiyoyama T, Tokuda Y, Shiiki S, Hachiman T, Shimasaki T, Endo K. Isopropyl alcohol compared with isopropyl alcohol plus povidone-iodine as skin preparation for prevention of blood culture contamination. J Clin Microbiol 2009;47(1):54-58.

\section{Healthcare Workers with Methicillin- Resistant Staphylococcus aureus Colonization and the Use of Contact Precautions in Daily Activities with Patients in an Italian Rehabilitation Hospital: The Importance of Hand Hygiene Training}

To the Editor-After reading with interest the article by Barbosa et al, ${ }^{1}$ which was published in the September 2009 issue of the journal, we would like to present the results of our point-prevalence study about methicillin-resistant Staphylococcus aureus (MRSA) colonization among healthcare workers (HCWs) of the Fondazione Santa Lucia - a rehabilitation hospital in Rome, Italy - and the use of contact precautions in daily activities with the patients of this hospital.

Our study was a part of the MOSAR (Mastering hOSpital Antimicrobial Resistance) project, work package 5. This project is a transdisciplinary network devoted to combating and controlling antimicrobial resistance of bacteria responsible for the major and emerging nosocomial infections that are now spreading into the community. MOSAR has 3 clinical work packages. Work package 5 (interventional trials in rehabilitation care units) involves 8 wards in 4 countries. The MOSAR project is coordinated by the French National Institute of Health and Medical Research, INSERM (Institut National de la Santé et de la Recherche Médicale), and supported by the European Commission under the Life Science Health Priority of the Sixth Framework Program.

Our point-prevalence study of MRSA colonization was performed during the period from June through July 2009 in 2 wards (with a mix of neurological, orthopedic, postcoma, and spinal cord injury cases) of the Fondazione Santa Lucia, which was already taking part in the MOSAR project. Our study was approved by the ethical committee and involved 129 of the 183 HCWs who were employed in the 2 wards and who belonged to different professions in the rehabilitation hospital (Table 1). Each HCW enrolled in the study was asked to complete a short questionnaire regarding their routine activities and use of contact precautions during daily activities (Table 1). A single-use cotton swab, immersed in sterile $0.9 \%$ $\mathrm{NaCl}$, was used to collect samples from the anterior vestibule of both nares of HCWs for culture.

Nasal swab samples were plated to a differential and MRSA-selective chromogenic medium (CHROMAgar; Becton Dickinson). Bacterial growth was evaluated at 24 and 48 hours for bacterial colonies belonging to $S$. aureus (ie, mauve color on CHROMAgar). Then all mauve-colored colonies were cultured on Columbia blood agar media (Oxoid), and MRSA colonies were confirmed by use of the Staphaurex latex agglutination test (Oxoid).

In our study, we found an overall MRSA colonization rate of $3.1 \%$. Among the 129 screened HCWs, only 4 nurses' aides tested positive for MRSA colonization. Nurses' aides in rehabilitation hospitals are responsible for taking care of patients on a daily basis. They assist in dressing, bathing, and feeding the patient. Many of their duties are conducted under the supervision of nurses. The nurses' aides with MRSA colonization were equally distributed over both wards. No MRSA colonization was found among other HCWs (eg, doctors, nurses, therapists, and other medical staff members such psychologists and dietitians).

MRSA colonization among HCWs is not routinely studied in Italian rehabilitation hospitals. However, our rates of MRSA colonization appear to be similar to those found in other studies of HCW colonization in Italy. Recent studies in Italy on a similar number of HCWs in a large teaching hospital $^{2}$ and a nursing home ${ }^{3}$ have reported MRSA colo-

TA B LE 1. Data on Use of Contact Precautions in Daily Activity and Colonization with Methicillin-resistant Staphylococcus aureus (MRSA) among Healthcare Workers (HCWs) at 2 Wards in an Italian Rehabilitation Hospital (June-July 2009)

\begin{tabular}{|c|c|c|c|c|c|c|}
\hline Variable & $\begin{array}{l}\text { Doctors } \\
(n=12)\end{array}$ & $\begin{array}{l}\text { Nurses } \\
(n=44)\end{array}$ & $\begin{array}{l}\text { Nurses' aides } \\
\quad(n=25)\end{array}$ & $\begin{array}{l}\text { Therapists } \\
(n=39)\end{array}$ & $\begin{array}{c}\text { Other } \\
\text { medical staff } \\
(n=9)\end{array}$ & $\begin{array}{c}\text { Total } \\
(n=129)\end{array}$ \\
\hline \multicolumn{7}{|c|}{ No. of HCWs who used contact precautions } \\
\hline All the time & 7 & 40 & 20 & 2 & 4 & 73 \\
\hline Sometimes & 5 & 3 & 5 & 25 & 3 & 41 \\
\hline None of the time & 0 & 1 & 0 & 12 & 2 & 15 \\
\hline \multicolumn{7}{|c|}{ No. of HCWs with nasal swab sample } \\
\hline positive for MRSA & 0 & 0 & 4 & 0 & 0 & 4 \\
\hline
\end{tabular}


nization rates of $1.5 \%$ and $5.8 \%$, respectively. HCWs' adherence with contact precautions was evaluated by use of a questionnaire (Table 1). Because no statistically significant differences were found among the different groups of HCWs (by use of the $\chi^{2}$ test), it can be supposed that the high rate of MRSA colonization in the nurses' aides group (16\%) is probably due to both misuse of gloves and poor compliance with correct procedures for hand hygiene, which can result in the transmission of MRSA.,5 The World Health Organization (WHO) multimodal programme "Clean Care is Safer Care," which was started in 2005, has been effective at reducing the spread of MRSA and has a central role to play in improving hygiene practices in hospitals. ${ }^{6}$

Since 2006, the Infection Control Committee at Fondazione Santa Lucia has implemented a hand hygiene improvement programme by introducing the technique of hand rubbing with an alcohol-based solution at the point of care and by monitoring hand hygiene compliance using repeated observational studies on care activities. As recommended by the WHO programme, we also developed training courses and guidelines on hand hygiene based on scientific evidence, and there are reminders in the workplace (eg, "patient partnership activity") that provide a climate of safety in the hospital. However, the above-mentioned educational activities were mainly directed at doctors, nurses, therapists, and other medical staff members, not at nurses' aides.

Our results suggest that, in the curricula of nurses' aides, more emphasis needs to be placed on hand hygiene training and on improving adherence to hand hygiene practice. In fact, in Italy, nurses' aides are supposed to receive 1,000 hours of educational training before they can work in a healthcare setting; however, only a few of those hours are devoted to understanding the WHO guidelines for hand hygiene and implementation surveillance tools. Furthermore, the lack of inclusion of nurses' aides in the Continuing Medical Education program may also prevent them from taking healthcare courses (on these very issues) that are normally organized for other professional HCWs. Because being a nurses' aide at Fondazione Santa Lucia is usually considered a permanent position, their inclusion in the Continuing Medical Education program (ie, in the next set of training and educational courses on hand hygiene) may result in a reduction in the incidence of MRSA colonization among HCWs in our hospital.

\section{ACKNOWLEDGMENTS}

Potential conflicts of interest. All authors report no conflicts of interest relevant to this article.

Angelo Rossini, MD, MPH; Maria Pia Balice, MSc; Ludovica Ciotoli, RN; Emilio Guaglianone, BSc; Gianfranco Donelli, PhD; Antonino Salvia, MD, MPH
From the Fondazione Santa Lucia, Istituto di Ricovero e Cura a Carattere Scientifico (A.R., M.P.B., L.C., E.G., G.D., A.S.), and the Istituto Superiore di Sanità (G.D.), Roma, Italy.

Address reprint requests to Angelo Rossini, MD, MPH, Fondazione Santa Lucia, Istituto di Ricovero e Cura a Carattere Scientifico, Via Ardeatina 306, Roma 00179, Italy (a.rossini@hsantalucia.it).

Infect Control Hosp Epidemiol 2010; 31(10):1097-1098

(C) 2010 by The Society for Healthcare Epidemiology of America. All rights reserved. 0899-823X/2010/3110-0025\$15.00. DOI: $10.1086 / 656380$

\section{REFERENCES}

1. Barbosa AA, Chapin K, Mermel LA. Methicillin-resistant Staphylococcus aureus colonization of house officers [published correction appears in Infect Control Hosp Epidemiol 2009;30(12):1242]. Infect Control Hosp Epidemiol 2009;30(9):912-914.

2. Orsi GB, Marrone R, Ferraro F, Tavella F, Colosi A. Low colonization with MRSA among health-care workers in an Italian hospital. Ann Ig 2008;20: 503-508.

3. Monaco M, Bombana E, Trezzi L, et al. Meticillin-resistant Staphylococcus aureus colonising residents and staff members in a nursing home in Northern Italy. $J$ Hosp Infect 2009;73:182-184.

4. Ludlam HA, Swayne RL, Kearns AM, et al. Evidence from a UK teaching hospital that MRSA is primarily transmitted by the hands of healthcare workers. J Hosp Infect 2010;74:296-299.

5. Girou E, Chai SH, Oppein F, et al. Misuse of gloves: the foundation for poor compliance with hand hygiene and potential for microbial transmission? J Hosp Infect 2004;57:162-169.

6. Allegranzi B, Pittet D. Role of hand hygiene in healthcare-associated infection prevention. J Hosp Infect 2009;73:305-315.

\section{Central Venous Catheter Flushing and an Outbreak of Bacteremia among Pediatric Hematology-Oncology Patients}

To the Editor-Wiersma et $\mathrm{al}^{1}$ reported an outbreak of polymicrobial bloodstream infections in pediatric bone marrow transplant outpatients. The investigation concluded that the preparation of multiple doses of predrawn saline flush solution from a $50-\mathrm{mL}$ single-dose saline vial, which was accessed multiple times, may have led to extrinsic contamination.

The central venous catheter (CVC) is an indispensable tool for the treatment of patients with cancer. ${ }^{2}$ One of the major drawbacks of the long-term use of a CVC is the development of CVC-related bacteremia, which increases morbidity, mortality, and healthcare costs. ${ }^{3} \mathrm{~A}$ breach of sterility is a common complication when there is an inappropriate modification of CVC procedures for handling, flushing, and delivering solutions and medications. We experienced an outbreak of CVC-related bacteremia among pediatric hematology-oncology patients caused by a change in the local policy for CVC flushing with heparin solution. In January 1999, there were 